\title{
An Auto-balancing Capacitance-to-Pulse-Width Converter for Capacitive Sensors
}

\author{
Lakshmi Areekath, Student Member, IEEE, Boby George, Senior Member, IEEE, and Ferran Reverter
}

\begin{abstract}
A novel auto-balancing capacitance-to-pulsewidth converter (CPC) that uses sinusoidal excitation, and operates in a closed-loop configuration, is presented in this paper. Unlike most of the existing CPCs, the proposed interface circuit is compatible with both single-element and differential capacitive sensors. In addition, it provides a pulse-width modulated (PWM) signal which can easily be digitized using a counter. From this PWM signal, a ratio output is derived when a single-element sensor is interfaced, and a ratiometric output is obtained for a differential sensor. The final digital output is independent of the nominal capacitance of the sensor and has a linear characteristic irrespective of the sensor characteristic being linear or inverse. The CPC is designed such that the PWM output depends on the change in the sensor capacitance alone. It is insensitive to parasitic capacitance and has very low sensitivity to the non-idealities of the components and ICs used. The effects due to some of the non-idealities are automatically corrected by the negative feedback based autobalancing employed. The effect of component mismatch is significantly reduced by a one-time correction mechanism. These benefits are achieved without the use of any complex or expensive analog building blocks. The prototype exhibits a maximum non-linearity error of less than $0.7 \%$, a resolution of 13.02 effective number of bits (ENOB), a signal-to-noise ratio (SNR) of $80.12 \mathrm{~dB}$, and a rise time of $5 \mathrm{~ms}$. Thus, the proposed simple, yet effective, low-power, low cost, auto-balancing CPC can be used to interface a wide range of existing and new capacitive sensors to digital systems.
\end{abstract}

Index Terms-Capacitive sensor, differential capacitive sensor, pulse-width converter, duty cycle, sine wave, autobalancing, digitizer, linearization, PWM, linear output, parasitic capacitance.

\section{Introduction}

$\mathrm{S}_{\mathrm{a}}^{\mathrm{E}}$ ENSORS with efficient interfacing circuits that are simple and reliable are in high demand in the world today. They play a key role in a variety of domains ranging from consumer electronics, agriculture, healthcare, to automation, industry, and scientific research [1]. The demand is a constantly growing one, driven by the popular technologies of data science and artificial intelligence, which are heavily dependent upon enormous amounts of useful sensor data. Among various types of sensors that are available, capacitive sensors are used in numerous applications, and have a noticeable share in the sensor market, due to their advantages such as ease of fabrication, high sensitivity, high resolution, low power dissipation, broad bandwidth, and low cost [1].

Depending on the application and requirement, measurement engineers opt for (a) single-element capacitive sensors, which are designed using two electrodes, (b) differential capacitive sensors (DCS), designed using three electrodes, or (c) sensors that use more electrodes, which can be considered as a combination of multiple single-element sensors [1]-[5]. The differential capacitive sensor has a wide range of applications in automation and control. Its output can be processed in a way that the effect due to most of the common parameters that are of no interest can be eliminated or reduced [1]. It is used not only to sense mechanical parameters such as position [2], displacement [3], acceleration [4], force [5], and shear stress [6], but also to realize proximity sensing [7], and nondestructive estimation [8]. These are also used in areas like gesture recognition [9], bacterial growth monitoring [10], robotic and upper limb prosthesis [6], pressure sensing in harsh environments [11], and accurate detection of a sound source [12]. The DCS consists of two sensing capacitances, $C_{1}$ and $C_{2}$, with a common electrode, as shown in Fig.1. The input-output characteristic of $C_{1}$ and $C_{2}$ can be linear, as in (1), or inverse, as shown in (2), where $k$ is a transformation constant and $C_{0}$ is the nominal capacitance of the sensor [13]. In the case of the ubiquitous parallel plate arrangement of the DCS, (1) holds true if the change in capacitance is caused by a change in the area of overlap between the plates, and (2) if the change is caused by the change in the distance between the plates. In the case of single-element capacitive sensors, $C_{1}$ in (1) can be used to represent the sensor with a linear characteristic, and similarly, $C_{2}$ in (2) can be used to represent the one following inverse characteristic.

$$
C_{1}=C_{0}(1 \pm k x) \text { and } C_{2}=C_{0}(1 \mp k x)
$$




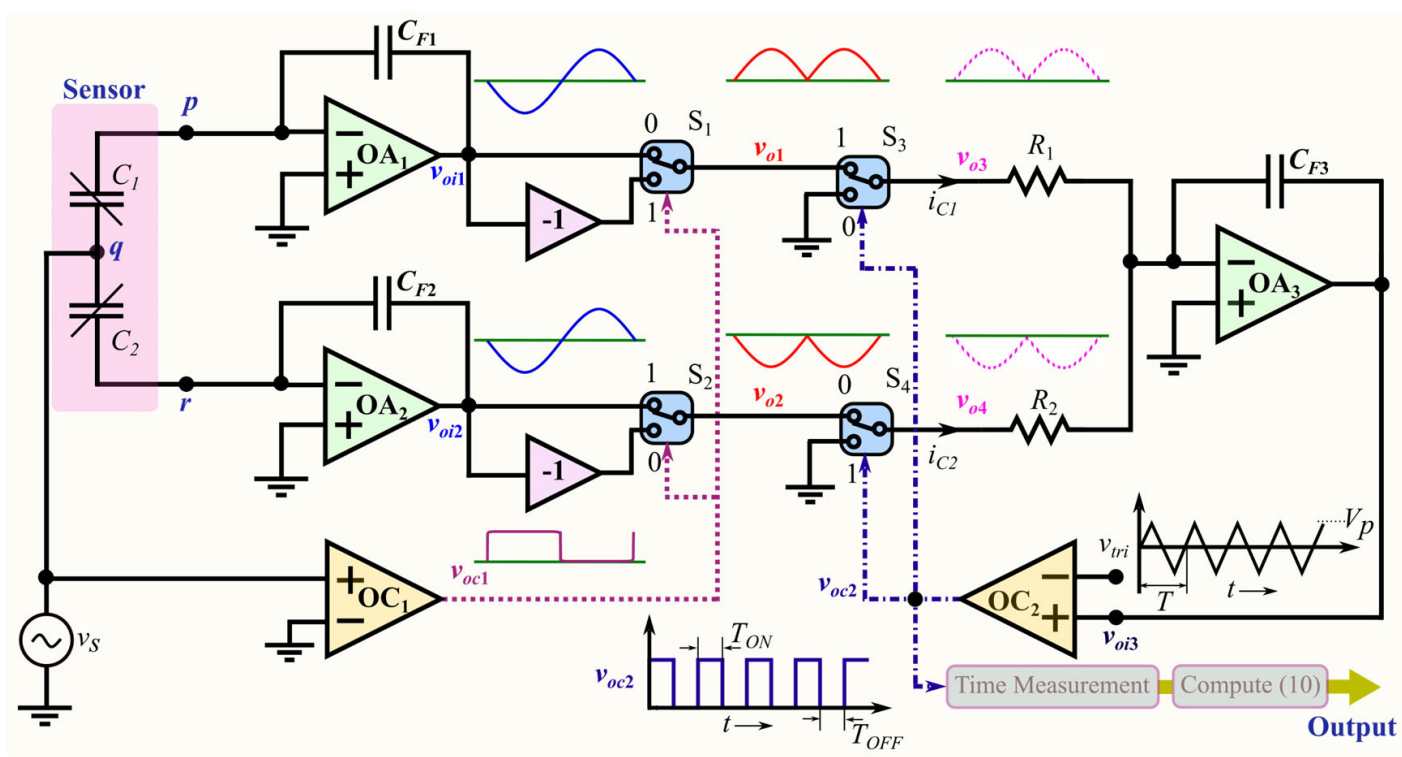

Fig. 1. Simplified circuit diagram of the proposed capacitance-to-pulse-width converter.

$$
C_{1}=\frac{C_{0}}{(1 \mp k x)} \text { and } C_{2}=\frac{C_{0}}{(1 \pm k x)}
$$

The conversion of the sensor capacitance to a form suitable for analog or digital signal processing can be done in many ways. It can be converted to an equivalent voltage [14], [15] or current [16]. In such a case, different techniques for compensating the effects of parasitic capacitances have been proposed, for instance: the use of a negative capacitance placed in parallel with the parasitic one [17], even including an automatic compensation circuit [18]. Capacitances-to-Digital converters (CDCs) based on voltage conversion, followed by an analog-to-digital converter, are generally complex in design, prone to noise and consume more power, compared to other conversion paradigms [19]. Interfacing schemes based on quasi-digital signals, where the information is given by either the frequency or pulse duration, have emerged as possible alternatives owing to their simplicity, the possibility of direct transmission over comparatively noisy or non-linear channels and the facility to digitize these signals using counters [19][22]. Another approach is to interface the capacitive sensor directly to a digital system such as a microcontroller, but the accuracy that can be achieved is limited even after employing special calibration [23]. Also, in this case, it gets relatively cumbersome if a sensor needs to be excited at a particular frequency. Capacitance-to-frequency converters (CFCs) are comparatively simpler and less power demanding circuits. However, their practical realizations are often fraught with high sensitivity to temperature and process parameters when used in an open-loop configuration [19]. Most of the CFCs require a voltage-to-frequency converter (VFC), which increases the cost and complexity of the interfacing circuit. Similar to the CFCs, the output of the capacitance-to-pulse-width converters (CPCs) can be easily interfaced to a microcontroller/digital system, but without the additional cost and complexity of analog blocks like the VFC. This is because the frequency of the PWM signal can be synchronized to that of the inbuilt clock of the microcontroller [22]. Also, the PWM signals, being quasidigital, possess all the advantages of a frequency output [19]. In spite of these attractive features, CPCs have not been widely explored so far. Although there are some CPCs reported for capacitive sensors excited from DC sources, there are no attempts of CPCs reported for differential capacitive sensors excited using a sinusoidal source. A basic idea of such a scheme for a single element capacitive sensor is presented in [24].

An important factor to consider in the design of the signal conditioning circuitry, for any capacitive sensor, is the type of excitation signal used. The sinusoidal AC signal is one such excitation signal. A sinusoidal signal is one of the best choices to evaluate a given system. Also, in the case of some of the capacitive sensors which use the change in the dielectric property with frequency [25], [26] the use of a sinusoidal excitation signal, within a specified frequency range, is unavoidable. This AC excitation signal has been used to successfully realize many sensing applications such as flow rate measurement [27], proximity sensing [28] and humidity measurement [29]. The use of this AC signal also places lesser demand on the opamps, used in the circuit, for required bandwidth and slew rate, in comparison with a square-wave signal. The use of such an excitation signal also enables the easy implementation of a synchronous demodulator to achieve interference insensitivity and high signal-to-noise ratio (SNR) [30].

In this paper, a novel auto-balancing CPC, with a sinusoidal excitation, which employs a new closed-loop pulse-width conversion technique, is presented. Its PWM output facilitates easy digitization of the interfaced sensor capacitance. This new scheme can be used to interface single-element as well as differential capacitive sensors. The sensors can possess either a linear characteristic as in (1), or an inverse one as in (2). The final output from the proposed CPC is linear. Thus, this scheme combines the use of a sinusoidal AC excitation, and provides a PWM signal, and so has several advantages, as mentioned above. Additionally, the proposed scheme is insensitive to 
parasitic capacitance. It also offers a solution to the degradation in performance of CDCs due to offset capacitance. Generally, the capacitive transducers used in practice produce very small changes due to the measurand. At the same time, they exhibit a relatively large offset capacitance. If not canceled, the offset also gets converted, and thus degrades the resolution of the capacitance measurement. The pulse-width converter presented in this paper processes the output corresponding to the change in capacitance alone, not the offset capacitance. In addition, the ratio output provided in the case of the single element sensor, and the ratiometric output obtained for the differential capacitive sensor are independent of the nominal capacitance $C_{0}$. These features are achieved without complex signal processing, which otherwise adds to the cost and power consumed.

\section{CApacitance-to-Pulse-Width CONVERTER}

The circuit diagram of the proposed CPC is shown in Fig. 1. Here, the variable capacitors $C_{1}$ and $C_{2}$ represent the differential capacitive sensor (DCS). The circuit is also suitable for singleelement capacitive sensors, which is discussed at the end of this section. The circuit uses a sinusoidal excitation signal $v_{S}=$ $\sqrt{2} V_{s} \sin \omega t$, where $V_{s}$ is the root mean square (rms) voltage, and $\omega=2 \pi f_{s}$, where $f_{s}$ is the frequency of the signal. Its time period $T_{s}=1 / f_{s}$. The maximum frequency at which $C_{1}$ and $C_{2}$ change is assumed to be much less compared to $f_{S}$. The opamps $\mathrm{OA}_{1}$ and $\mathrm{OA}_{2}$, feedback capacitors $C_{F 1}$ and $C_{F 2}$, single-pole, double throw (SPDT) switches $\mathrm{S}_{1}, \mathrm{~S}_{2}, \mathrm{~S}_{3}$ and $\mathrm{S}_{4}$, and resistors $R_{1}$ and $R_{2}$, form two branches of the proposed circuit, beginning at $C_{1}$ and at $C_{2}$, respectively, and converging at the inverting terminal of opamp $\mathrm{OA}_{3} . \mathrm{OA}_{1}, C_{F 1}$, and $C_{1}$ constitute a charge amplifier. So do $\mathrm{OA}_{2}, C_{F 2}$, and $C_{2}$. The outputs $v_{o i 1}$ and $v_{o i 2}$ of these two charge amplifiers are expressed as, $v_{o i 1}=$ $-\frac{C_{1}}{C_{F 1}} \sqrt{2} V_{s} \sin \omega t$ and $v_{o i 2}=-\frac{C_{2}}{C_{F 2}} \sqrt{2} V_{S} \sin \omega t$

As shown in Fig. 1, $v_{o i 1}$ and $v_{o i 2}$ are processed using $\mathrm{S}_{1}$ and $\mathrm{S}_{2}$, respectively, and two unidirectional signals of opposite polarity, $v_{o 1}$ and $v_{o 2}$, respectively, are obtained. Both $\mathrm{S}_{1}$ and $\mathrm{S}_{2}$ are controlled by the output $v_{o c 1}$ of the comparator $\mathrm{OC}_{1}$. The inputs of $\mathrm{OC}_{1}$ are $v_{S}$ at the non-inverting terminal, and ground potential at the inverting terminal. Thus, $v_{o c 1}$ is a square wave of frequency $f_{s}$. When $v_{o c 1}$ is low, $\mathrm{S}_{1}$ and $\mathrm{S}_{2}$ are at position- 0 leading to $v_{o 1}=v_{o i 1}$ and $v_{o 2}=-v_{o i 2}$. Alternatively, when $v_{o c 1}$ is high, both $\mathrm{S}_{1}$ and $\mathrm{S}_{2}$ are at position-1, and so $v_{o 1}$ and $v_{o 2}$ are $-v_{o i 1}$ and $v_{o i 2}$, respectively. A pictorial representation of these signals is included in Fig. 1. These signals are fed into $S_{3}$ and $\mathrm{S}_{4}$, respectively. Both $\mathrm{S}_{3}$ and $\mathrm{S}_{4}$ are controlled by $v_{o c 2}$, a pulse signal generated by the comparator $\mathrm{OC}_{2}$. The signal at the output of $\mathrm{S}_{3}, v_{o 3}=v_{o 1}$ when $v_{o c 2}$ is high, and zero (ground) otherwise. The opposite occurs at the output of $\mathrm{S}_{4}$, with $v_{04}=0$ when $v_{o c 2}$ is high, and $v_{o 4}=v_{o 2}$ otherwise. $v_{o 3}$ and $v_{o 4}$ are input via $R_{1}$ and $R_{2}$, respectively, into the inverting terminal of $\mathrm{OA}_{3} . R_{1}, R_{2}$, and $\mathrm{OA}_{3}$, along with the feedback capacitor $C_{F 3}$ constitute an inverting summing integrator. Its output, $v_{o i 3}$, is given to the non-inverting terminal of $\mathrm{OC}_{2}$. A reference triangular signal $v_{t r i}$, with peak value $V_{p}$ and time period $T=$ $1 / f_{\text {tri }}$, is given to its inverting terminal.

The pulse-width of $v_{o c 2}$ depends on $v_{o i 3}$ and $v_{t r i} . v_{o c 2}$ is high while $v_{t r i}<v_{o i 3}$ and low otherwise. In the proffered circuit $f_{t r i}$ is set such that $f_{t r i} \gg f_{S}$ (explained in section II.A.). Hence, the change in $v_{o i 3}$ within a cycle of $v_{t r i}$ is very low compared to that in $v_{t r i}$. When $v_{o i 3}=0$, the pulse-width of $v_{o c 2}$ is $T / 2$, and the duty cycle $D=\frac{T_{O N}}{T}$ is 0.5 . Here, $T_{O N}$ is the duration for which $v_{O c 2}$ is high. $T_{O F F}$ is the duration for which $v_{o c 2}$ is low and thus, $T=T_{O N}+T_{O F F}$. As $v_{o i 3}$ increases in the positive direction, $T_{O N}$ increases, and $T_{O F F}$ decreases. The opposite occurs $\left(T_{O N}\right.$ decreases and $T_{O F F}$ increases) if $v_{o i 3}$ decreases from zero and goes negative. The control of $\mathrm{S}_{3}$ and $\mathrm{S}_{4}$ by $v_{o c 2}$, whose pulsewidth is a function of $v_{\text {ois }}$, realizes a negative feedback based the auto-balancing mechanism in the proposed circuit. The operation of the proposed CPC when interfaced with (a) differential, and (b) single-element capacitive sensors, respectively, is discussed below.

\section{A. Differential Capacitive Sensor}

In the proposed circuit, $C_{F 1}=C_{F 2}=C_{F}$ and $R_{1}=R_{2}=R$. The output voltages $v_{o 1}$ and $v_{o 2}$ of the switches $\mathrm{S}_{1}$ and $\mathrm{S}_{2}$, respectively, can be expressed as $v_{o 1}=\left|\frac{C_{1}}{C_{F}} \sqrt{2} V_{s} \sin \omega t\right|$ and $v_{o 2}=-\left|\frac{C_{2}}{C_{F}} \sqrt{2} V_{S} \sin \omega t\right|$. As $\mathrm{S}_{3}$ is controlled by $v_{o c 2}, v_{o 3}=v_{o 1}$ during $T_{O N}(=D T)$, but zero for $T_{O F F}$. The average value, $\overline{l_{C 1}}$, of current $i_{C 1}$ flowing through $R_{1}$, as shown in Fig. 1, for one cycle of $v_{S}$ is expressed as in (3). In (3), $\left(T_{s} / T\right)$ is a positive integer.

$$
\overline{l_{C 1}}=\frac{1}{T_{S}} \sum_{j=0}^{j=\left(\frac{T_{S}}{T}-1\right)} \int_{j T}^{D T+j T}\left|\frac{C_{1}}{C_{F} R} \sqrt{2} V_{S} \sin \omega t\right| d t
$$

Similarly, the average value, $\overline{l_{C 2}}$, of current $i_{C 2}$ flowing through $R_{2}$, for one cycle of $v_{S}$ is expressed as in (4). This is written based on $v_{O 4}=v_{O 2}$ during $T_{O F F}$, i.e. $(1-D) T$, and zero otherwise.

$$
\overline{l_{C 2}}=\frac{1}{T_{S}} \sum_{j=0}^{j=\left(\frac{T_{S}}{T}-1\right)} \int_{D T+j T}^{T+j T}-\left|\frac{C_{2}}{C_{F} R} \sqrt{2} V_{S} \sin \omega t\right| d t
$$

From (3) and (4), $\overline{l_{C 1}}$ and $\overline{l_{C 2}}$ are directly proportional to $C_{1}$ and $C_{2}$, respectively. Consider the case $D=50 \% . v_{o 3}=v_{o 1}$ when $v_{c 2}$ is high, and zero otherwise. Similarly, $v_{o 4}=v_{o 2}$ when $v_{c 2}$ is low, and zero otherwise. These are illustrated in Fig. 2. If the top portions of each non-zero segment of $v_{o 3}$ and $v_{04}$ are assumed to be flat, the segments become rectangular blocks. As

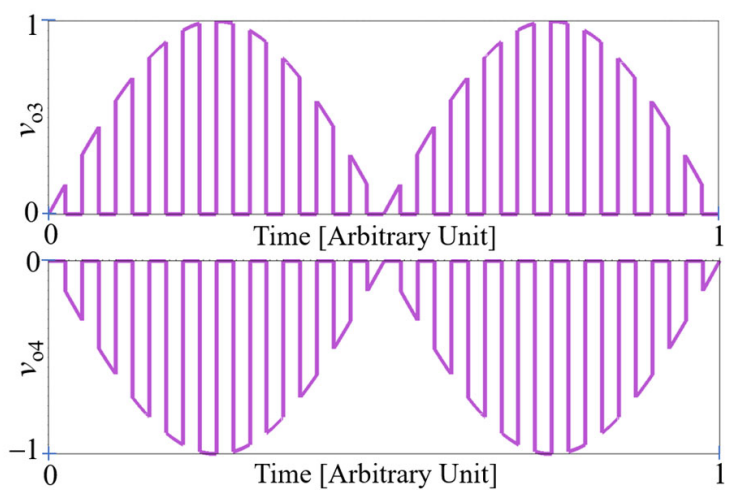

Fig. 2. Signals $v_{03}$ and $v_{04}$ when $D=0.5$. This is illustrated for $f_{t r i}=20 f_{S}$. The sum of the average of these signals was found to be negligible $\left(0.006 \%\right.$ of $\left.\overline{v_{o 4}}\right)$. The computation was done using Scilab. 
long as $T_{S} \gg T$, this approximation will introduce only a negligible error and the average value of the signal can be computed using these rectangular blocks. Under this condition, the average value of $v_{03}$ will vary proportional to $D$, and that of $v_{o 4}$ proportional to $(1-D)$. Thus, the values of $\overline{l_{C 1}}$ computed using (3) and $\overline{l_{C 2}}$ computed using (4) are directly proportional to $D$ and $(1-D)$, respectively. The relationship between $\overline{l_{C 1}}$ and $D$ is shown in Fig. 3, which is computed by varying $D$ while maintaining $C_{1}$ at a fixed value. It can be seen that the curve becomes linear once the condition $T_{S} \gg T$ is satisfied. The nonlinearity error becomes negligible once $f_{t r i}$ is $20 f_{s}$ or more. The same can be concluded in case of $\overline{l_{C 2}}$ and $(1-D)$.

The integrator output voltage $v_{o i 3}$ can be expressed as in (5).

$$
v_{o i 3}=-\frac{1}{C_{F 3}} \int_{0}^{T_{S}}\left(i_{C 1}+i_{C 2}\right) d t
$$

When $C_{1}=C_{2}, \overline{l_{C 1}}=-\overline{l_{C 2}}$ and from (5), $v_{o i 3}=0$. Then, $T_{O N}=T_{O F F}$ and $D=50 \%$. In case $C_{1}<C_{2}$, initially $\left|\overline{l_{C 1}}\right|<$ $\left|\overline{l_{C 2}}\right|$, as per (3) and (4). As $\overline{l_{C 2}}$ is a negative quantity, $v_{o i 3}$ will increase in the positive direction. This causes $T_{O N}$ to increase and $T_{O F F}$ to decrease, increasing $D$. This will increase $\bar{l}_{C 1}$ and decrease $\bar{l}_{C 2}$. This process continues until $v_{o i 3}$ reaches the steady state, i.e., $\overline{l_{C 1}}=-\overline{l_{C 2}}$. The opposite happens (i.e., $\left|\overline{l_{C 1}}\right|>\left|\overline{l_{C 2}}\right|$ and $v_{o i 3}$ will increase in the negative direction) when $C_{1}>C_{2}$.

In the steady state condition, i.e., when $\frac{d\left(v_{\text {ois }}\right)}{d t}=0, i_{C 1}$ and $i_{C 2}$ are related as in (6).

$$
\overline{l_{C 1}}=-\overline{l_{C 2}}
$$

Considering $\overline{l_{C 1}}$ is directly proportional to $C_{1}$ and $D$, and $\overline{l_{C 2}}$ to $C_{2}$ and $(1-D),(6)$ can be rewritten as in (7).

$$
C_{1} \times D=C_{2} \times(1-D)
$$

Rearranging $C_{1}$ and $C_{2}$ and expressing (7) in terms of $T_{O N}$ and $T_{O F F}$, gives (8).

$$
\frac{C_{1}}{C_{2}}=\frac{T_{O F F}}{T_{O N}}
$$

Further, in ratiometric form, (8) becomes

$$
\frac{C_{1}-C_{2}}{C_{1}+C_{2}}=\frac{T_{O F F}-T_{O N}}{T_{O F F}+T_{O N}}
$$

By substituting for $C_{1}$ and $C_{2}$ as in either (1) or (2), i.e., sensors with either linear or inverse characteristic, respectively, (9) becomes (10). As can be seen from (10), the final output of the proposed scheme is linear, irrespective of the sensor characteristic being linear or inverse.

$$
\pm k x=\frac{T_{O F F}-T_{O N}}{T_{O F F}+T_{O N}}
$$

\section{B. Single-Element Capacitive Sensor}

In addition to being compatible with differential capacitive transducers, the proffered CPC is also suitable for singleelement capacitive sensors. To interface a single-element capacitive sensor, either $C_{1}$ or $C_{2}$, shown in Fig. 1, is set as the variable capacitance of sensor, $C_{x}$, while the other acts as a fixed reference capacitance, $C_{R}=C_{0}$. The choice of either $C_{1}$ or $C_{2}$ as $C_{x}$ depends upon the sensor characteristic of $C_{x}$ : (a) if

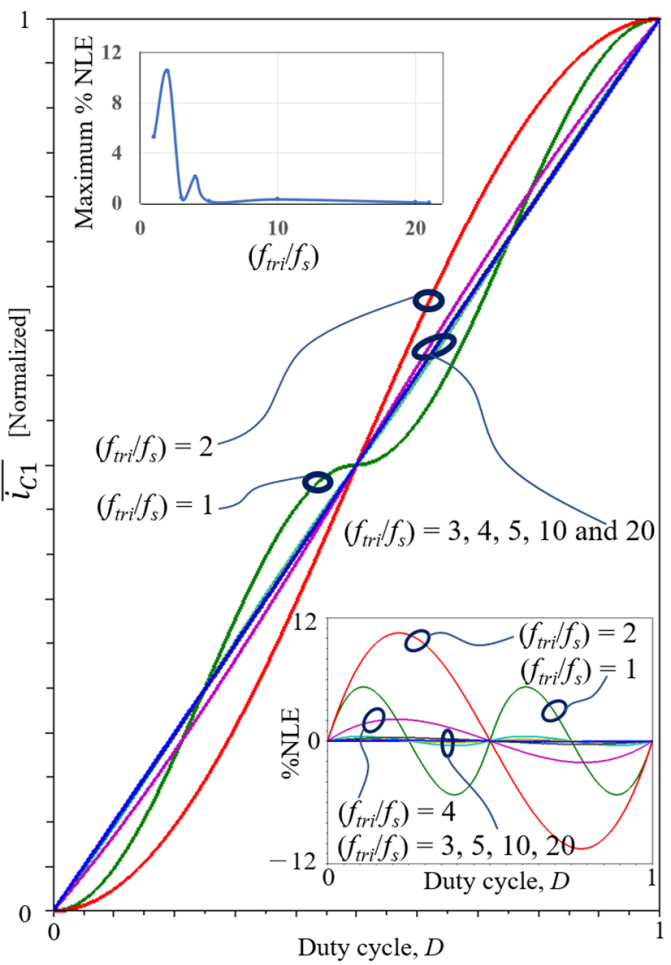

Fig. 3. The average value of the signal $i_{C 1}, \overline{l_{C 1}}$, for different values of $D$, from 0 to 1 . This was computed using Scilab, for $\left(f_{\text {tri }} / f_{s}\right)=1,2$, $3,4,5,10$, and 20 . The percentage non-linearity error (\%NLE) for all the corresponding ratios of $\left(f_{\text {tri }} / f_{s}\right)$ are shown in the bottom inset. The maximum of the $\% \mathrm{NLE}$, for the same ratios are given in the top left inset. It also gives the \%NLE for $\left(f_{\text {tri }} / f_{s}\right)=21$. It can be seen that the $\%$ NLE is very low as long as the ratio is high, say 20 .

$C_{x}$ possesses a linear characteristic, i.e. $C_{x}=C_{0}(1 \pm k x)$, then $C_{1}$ is set as $C_{x}$ and $C_{2}$ is set as $C_{0}$, and (b) if $C_{x}$ follows an inverse characteristic, i.e., $C_{x}=C_{0} /(1 \pm k x)$, then $C_{2}$ is set as $C_{x}$ and $C_{1}=C_{0}$. Substituting either of the choices (a) or (b) in (8) leads to (11).

$$
\pm k x=\frac{\left(T_{O F F}-T_{O N}\right)}{T_{O N}}
$$

According to (11), when a single-element capacitive sensor $C_{x}$ is interfaced to the proposed CPC, the final output is linear irrespective of its sensor characteristic.

\section{ANALYSIS: EFFECT OF NON-IDEALITIES}

\section{A. Component Mismatch - One time Correction}

The derivation of (10) has been done assuming $C_{F 1}=C_{F 2}=$ $C_{F}$, and $R_{1}=R_{2}=R$. In a practical implementation of the proposed scheme, the match in the aforementioned capacitors and resistors will be limited by the tolerance of these components. For example, let $C_{F 2}=C_{F 1}\left(1+\epsilon_{F}\right)$, where $\epsilon_{F}=$ $\frac{\Delta C_{F 1}}{C_{F 1}}$, and $\Delta C_{F 1}=C_{F 2}-C_{F 1}$. Then, in the steady state condition $\left|\overline{l_{C 1}}\right|=-\left|\overline{l_{C 2}}\right|,(7)$ gets modified as in (12).

$$
\left(\frac{1}{C_{F 1}}\right) C_{1} \times D=\left(\frac{1}{C_{F 2}}\right) C_{2} \times(1-D)
$$


Substituting $C_{F 2}=C_{F 1}\left(1+\epsilon_{F}\right)$ and $D=T_{O N} /\left(T_{O N}+T_{O F F}\right)$, and rearranging, gives (13), where $k_{F}=1 /\left(1+\epsilon_{F}\right)$.

$$
\frac{C_{1}}{C_{2}}=k_{F} \times \frac{T_{O F F}}{T_{O N}}
$$

The impact of $k_{F}$ in the final output can be compensated for by performing a simple one-time calibration. For this, $C_{1}$ and $C_{2}$ are set to their nominal value $C_{0}$. In the presence of mismatch $T_{O N}$ and $T_{O F F}$ will not be equal. Then, the value of $k_{F}$ can be obtained as $k_{F}=T_{O N} / T_{O F F}$. From then onwards, the measured $T_{O F F(m)}$ can be divided by $k_{F}$ to get the gain corrected $T_{O F F}$. This can be used in the final ratiometric output in (10) or ratio output in (11) to ensure negligible sensitivity to capacitor mismatch. A mismatch between $R_{1}$ and $R_{2}$ will result in a similar gain error. The $k_{F}$ obtained through the one-time calibration, is one that can be used for the gain correction of the signal paths (a) $C_{F 1}-\mathrm{S}_{3}-\mathrm{S}_{1}-R_{1}$ and (b) $C_{F 2}-\mathrm{S}_{2}-\mathrm{S}_{4}-R_{2}$ of the circuit in Fig. 1 .

\section{B. Parasitic Capacitances of the Transducer}

The parasitic capacitances $C_{P 1}, C_{P 2}$ and $C_{P 3}$ of the differential capacitive transducer are shown in Fig. 4. $C_{P 1}$ and $C_{P 2}$ remain inactive because in both of them one end is connected to the virtual ground while the other is grounded. $C_{P 3}$ has no impact on the output of the proposed circuit as long as it does not load the excitation source due to the current $I_{P 3}$ from $v_{s}$ to ground, through $C_{P 3}$.

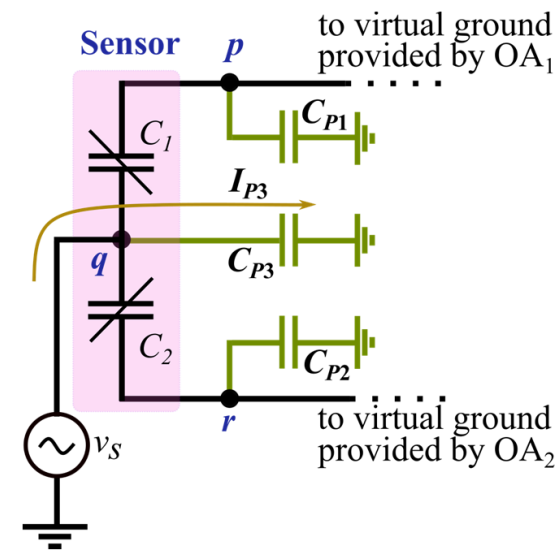

Fig. 4. Sensor with parasitic capacitances in the proposed CPC.

\section{Input Offset Voltage of Comparators}

Let $V_{O S C 1}$ and $V_{O S C 2}$ be the input offset voltages of $\mathrm{OC}_{1}$ and $\mathrm{OC}_{2}$, respectively. If the polarity of $V_{O S C 1}$ is such that the output $v_{O C 1}$ is high when the input signal to $\mathrm{OC}_{1}$ is zero, then, the duration for which $v_{O C 1}$ is high within a cycle of $v_{S}$ increases, and that for which it is low decreases. This will affect the switching of $\mathrm{S}_{1}$ and $\mathrm{S}_{2}$. In $v_{o 1}$, there will be small portions of negative voltage near each of the zero-crossing points of $v_{\text {oi1 }}$. The average value of this negative voltage within a cycle $T_{S}$ is $\cong \frac{V_{O S C 1}^{2}}{8 V_{S}^{2}} v_{o i 1(\max )}$, when $v_{S}$ and $v_{o i 1}$ are approximated to triangular waveforms (considering $V_{O S C 1}$ is very low compared to the peaks of the signals $v_{S}$ and $v_{o i 1}$ ), instead of sinewaves. $v_{o i 1(\max )}=\sqrt{2} V_{s} \frac{C_{1}}{C_{F}} \cdot v_{o i 1(\max )}$ can be designed such that it is close to $V_{S A T}-0.5$, where $V_{S A T}$ is the saturation voltage of $\mathrm{OA}_{1}$. For a comparator with a significant input offset voltage of 5 $\mathrm{mV}, \quad V_{S}=2.5 / \sqrt{2} \quad \mathrm{~V}, \quad$ and $\quad V_{S A T}=8 \quad \mathrm{~V}$, and $v_{\text {oi1 (max) }}=0.5\left(V_{S A T}-0.5\right)$, the resulting average value is $3.75 \mu \mathrm{V}$, which is negligible compared to the average value of $v_{o 1} \cong 2\left(V_{S A T}-0.5\right) / \pi=4.7 \mathrm{~V}$ at full scale. The same logic can be extended to quantify the effect of $V_{O S C 1}$ on the output of switch $\mathrm{S}_{2}$, and arrive at the conclusion that it can be neglected.

To understand the effect of $V_{O S C 2}$, consider first the case $C_{1}=$ $C_{2}$. In this condition, ideally, i.e. $V_{O S C 2}=0, v_{o i 3}$ should be zero and $T_{O N}=T_{O F F}$. However, due to $V_{O S C 2}$, when $v_{o i 3}=0, v_{O C 2}$ will not have transitions at the erstwhile zero crossing points of $v_{\text {tri }}$. Instead, the transitions occur when the value of $v_{\text {tri }}$ crosses $V_{O S C 2}$. If the polarity of $V_{O S C 2}$ is such that $v_{O C 2}$ is high when $v_{o i 3}=v_{t r i}=0$, then $T_{O N}>T_{O F F}$ and $\left|\overline{l_{C 1}}\right|>\left|\overline{l_{C 2}}\right|$. This will start driving $v_{o i 3}$ in the negative direction and the difference between $T_{O N}$ and $T_{O F F}$ will keep reducing with time. This process will stop when $v_{o i 3}$ has the same magnitude of that of $V_{O S C 2}$ but opposite polarity, ensuring $T_{O N}=T_{O F F}$. The impact of $V_{O S C 2}$ with opposite polarity can be analyzed in the similar manner. Thus, thanks to the negative feedback approach adopted in the proposed auto-balancing pulse-width converter circuit, $V_{O S C 2}$ has no effect in the final output.

\section{Bias Currents and Input Offset Voltage of Opamps}

Let $I_{B 1}, I_{B 2}$ and $I_{B 3}$ be the bias currents at the inverting terminals of $\mathrm{OA}_{1}, \mathrm{OA}_{2}$ and $\mathrm{OA}_{3}$, respectively. $I_{B 1}$ and $I_{B 2}$ charge $C_{F 1}$ and $C_{F 2}$, respectively, in a continuous manner. As in the case of charge amplifiers, to avoid driving the opamp into saturation, appropriately chosen large resistors are connected in parallel to $C_{F 1}$ and $C_{F 2}$, respectively, to provide a discharging path for each. The value of bias current is very low for commercially available opamps. Thus, the effect can be neglected, once the integrating effect is compensated using suitable parallel resistors.

$\mathrm{OA}_{3}$ is in the negative feedback loop of the auto-balancing pulse width converter. It does not require a parallel resistor as the feedback will regulate the integrating effect due to $I_{B 3}$. In the presence of $I_{B 3}, v_{o i 3}$ will have an integrating term $\frac{1}{C_{F 3}} \int I_{B 3} d t$. Due to this, to reach steady state, an additional current (equal and opposite to $I_{B 3}$ ) will have to be provided by $\overline{l_{C 1}}$ and $\overline{l_{C 2}}$. At steady state, (6) will get modified to become (14), instead of being $\overline{l_{C 1}}+\overline{l_{C 2}}=0$.

$$
\overline{l_{C 1}}+\overline{l_{C 2}}=I_{B 3}
$$

If we consider the condition $C_{1}=C_{2}, T_{O N}$ and $T_{O F F}$ will get modified as $T_{O N} \mp \Delta T_{\varepsilon}$ and $T_{O F F} \pm \Delta T_{\varepsilon}$ to contribute the opposite current that amounts to $I_{B 3}$, through $C_{F 3} . \Delta T_{\varepsilon}$ is the change in the ON and OFF periods due to $I_{B 3}$. This is equivalent to having an ideal opamp $\mathrm{OA}_{3}$ but with $C_{1}$ a bit greater than $C_{2}$. The effect of $I_{B 3}$ can be treated as an offset in the final output, and (10) will get modified as (15). It can be corrected digitally in the measured ON and OFF periods of $v_{O C 2}$. To keep the term $\frac{2 \Delta T_{\varepsilon}}{T_{O F F}+T_{O N}}$ very low, an opamp with very low bias current compared to $i_{C 1}$ and $i_{C 2}$ is preferred. 


$$
\pm k x=\frac{T_{O F F}-T_{O N}}{T_{O F F}+T_{O N}} \mp \frac{2 \Delta T_{\varepsilon}}{T_{O F F}+T_{O N}}
$$

Let $V_{O S 1}, V_{O S 2}$, and $V_{O S 3}$ be the input offset voltages of $\mathrm{OA}_{1}$, $\mathrm{OA}_{2}$ and $\mathrm{OA}_{3}$, respectively. $V_{O S 1}$ becomes present at the output of $\mathrm{OA}_{1}$ and, therefore, $v_{o 1}=\left(\left|v_{o i 1}\right| \mp V_{O S 1}\right)$ during the first half cycle, and $v_{o 1}=\left(\left|v_{o i 1}\right| \pm V_{O S 1}\right)$ in the second; this is assuming that the circuit is not in the initial transient after being supplied and, hence, the offset is not affected by the charge amplifier gain. Thus, the average value of the signal $v_{o 1}$ over one full cycle of $v_{s}$ will not have any effect due to $V_{O S 1}$. Hence, the final output will not be affected due to $V_{o s 1}$. Similarly, it can be seen that the effect of $V_{O S 2}$ on $v_{O 2}$, and thereby the final output is negligible.

Due to $V_{O S 3}$ there will be a fixed term $V_{O S 3}$ in $v_{o i 3}$. The effect of this will get cancelled as in the case of $V_{O S C 2}$, thanks to the negative feedback in the auto-balancing approach. In addition, there will be a current $V_{O S 3} / R_{E}$ flowing through $C_{F 3}$, introducing an additional integrating term $\frac{1}{C_{F 3}} \int\left(\frac{V_{O S 3}}{R_{E}}\right) d t$ in $v_{o i 3}$, where $R_{E}=R / 2$. This is equivalent to the case of $I_{B 3}$. The effect can be analyzed by replacing $I_{B 3}$ in (14) with $\frac{V_{O S 3}}{R_{E}}$. The final output will modify as in (15). Again, to keep the term $2 \Delta T_{\varepsilon}$ in (15) extremely low compared to $T_{O N}+T_{O F F}$, an opamp with very low input offset voltage that drives negligible current $\frac{V_{O S 3}}{R_{E}}$ compared to $i_{C 1}$ and $i_{C 2}$ needs to be selected and used.

\section{E. Non-idealities of the Inverting Amplifiers}

The above explanation assumed ideal inverting amplifiers, with gain -1 , feeding to the switches $S_{1}$ and $S_{2}$ as in Fig. 1 . The inverting amplifier processing $v_{o i 1}$ will provide an output $-v_{o i 1}\left(1+\epsilon_{R_{1}}\right)$, where $\epsilon_{R_{1}}$ is a gain error due to the mismatch between the feedback and input resistors. Similarly, the one processing $v_{o i 2}$ will give $-v_{o i 2}\left(1+\epsilon_{R_{2}}\right)$, where $\epsilon_{R_{2}}$ is again a gain error due to the resistors mismatch. These gain errors will be present throughout a half cycle of $v_{o 1}$ and $v_{o 2}$. The effect, in the final output, will automatically get corrected once the onetime correction for the mismatch, between the signal paths, is implemented as described in section III.A.

Also, the inverting amplifier will have a very small dc output that is twice the input offset voltage of the opamp. An opamp with very low input offset voltage $(75 \mu \mathrm{V})$ is selected such that this value is negligible compared to average values of the switch outputs $v_{O 1}$ and $v_{O 2}$ corresponding to the lowest range of the capacitive sensor. Additionally, there will be a dc part in the inverting amplifier output due to the bias current. To keep this value very low, the appropriate value of the resistor was introduced in the non-inverting terminal of the opamp [30].

\section{F. Non-idealities of the Switches}

Two main factors of the switch that need to be analyzed are charge injection, and ON resistance, $r_{O N}$. Switches with zero charge injection are available, e.g., MAX4709, hence the first factor can easily be taken care of. (6), and then (10) and (11) have been derived assuming that the equivalent resistance seen from the input of $\mathrm{OA}_{3}$ is same for both the signal paths (i) with $R_{1}, \mathrm{~S}_{3}$ and $\mathrm{S}_{1}$, and (ii) with $R_{2}, \mathrm{~S}_{4}$, and $\mathrm{S}_{2}$. In the circuit, $R_{1}=R_{2}=R$. Hence switches with identical ON resistance is preferred. Since the durations for which $\mathrm{S}_{3}$ and $\mathrm{S}_{4}$ are respectively connected to the corresponding input signals are not the same, when $C_{1} \neq C_{2}$ the effective $\mathrm{ON}$ resistance of the switches, for those two channels (i) and (ii), will be a function of $T_{O N}$ and $T_{O F F}$. Thus, the best way to limit the effect of $r_{O N}$ is by selecting switches that have $r_{O N}<<R$.

Each switch takes a finite delay time $t_{d}$ for every transition in its output. For the switch used in the prototype, $t_{d}$ is a few hundreds of nanoseconds which is a factor of 1000 times less than the time period $T$ of $v_{t r i}$. It is important that $t_{d} \ll T$. Since the same delay is present for both low-to-high and high-to-low transitions, the width of the $v_{03}$ and $v_{04}$ waveforms (refer Fig. 2) remains the same except that there will be a finite delay in each transition. In addition, switches $\mathrm{S}_{3}$ and $\mathrm{S}_{4}$ are controlled by the same signal and, hence, there will be no error as long as the mismatch between $t_{d}$ for both the switches is negligible. As both $\mathrm{S}_{3}$ and $\mathrm{S}_{4}$ have the same value $\left(R_{1}=R_{2}\right)$ of the resistor connected at the common terminal, the mismatch in $t_{d}$ is expected to be negligible. On the other hand, the delay time will have no noticeable error for $S_{1}$ and $S_{2}$ as it is much smaller than $T_{S}$ (= $\left.2 \mathrm{~ms}\right)$. Also, for $\mathrm{S}_{1}$ and $\mathrm{S}_{2}$ the value of the signal during the transition is very close to zero. The delay time due to comparator $\mathrm{OC}_{1}$ will have the same effect as that due to $t_{d}$ of $\mathrm{S}_{1}$ and $\mathrm{S}_{2}$. Similarly, the delay time of $\mathrm{OC}_{2}$ can be treated as an additional delay time in the transitions of $\mathrm{S}_{3}$ and $\mathrm{S}_{4}$.

Another limitation of the switches is the OFF resistance of the non-selected input. When $S_{1}$ is at position-0, as voltages at node- 0 and node- 1 are in phase opposition, there will be a small current between these nodes through the OFF resistance. This current will not go to the next stage through the common terminal of the switch. The same logic applies to the switch $\mathrm{S}_{2}$. On the other hand, when $S_{3}$ is at position-1, there will be a small current flowing from node- 1 to node- 0 . This value will be very low as $R_{1}=100 \mathrm{k} \Omega$ is much less than the OFF resistance. Due to this, there will be a small gain error in this signal path. A similar effect is present in the case of $\mathrm{S}_{4}$. The mismatch between these gain errors will be corrected in the one-time mismatch correction described in section III.A.

\section{G. Saturation of Opamps}

Let $\pm V_{S A T}$ be the positive/negative saturation voltage of both $\mathrm{OA}_{1}$ and $\mathrm{OA}_{2}$. In order to avoid saturation problems, the following condition must be satisfied: $-V_{S A T}<v_{o i j}<+V_{S A T}$, where $j$ can be either 1 or 2 . Assuming the expressions of $v_{o i j}$ given in Section II, the previous condition implies that $C_{j}<$ $V_{S A T} C_{F j} / \sqrt{2} V_{s}$. Accordingly, for a sensor with a linear and inverse characteristic, the range of $k x$ is limited as indicated in (16) and (17), respectively.

$$
\begin{aligned}
& |k x|<\frac{V_{S A T}}{\sqrt{2} V_{S}} \frac{C_{F j}}{C_{0}}-1 \\
& |k x|<1-\frac{\sqrt{2} V_{S}}{V_{S A T}} \frac{C_{0}}{C_{F j}}
\end{aligned}
$$

Considering the value of the components employed later in Section IV, the proposed circuit provides a full range operation (i.e., $-1 \leq k x \leq 1$ ) for the linear case, but not completely for the inverse case, to be precise: $-0.92 \leq k x \leq 0.92$. 


\section{H. Summary}

As a brief summary of the analysis, the important parameters that are considered in the analysis and their effect or the condition to keep the associated error within the limit are tabulated and presented in Table I.

TABLE I

CIRCUIT PARAMETERS AND THEIR EFFECT

\begin{tabular}{cc|cc}
\multicolumn{3}{c}{ CIRCUIT PARAMETERS AND THEIR EFFECT } \\
\hline \hline Source & Effect/remedy & Source & Effect/remedy \\
\hline $\begin{array}{c}\text { Component } \\
\text { mismatch } \\
\text { Parasitic } \\
\text { capacitance }\end{array}$ & $\begin{array}{c}\text { One-time } \\
\text { correction. }\end{array}$ & $\begin{array}{c}\text { ron, OFF } \\
\text { resistance }\end{array}$ & $\begin{array}{c}\text { One-time } \\
\text { correction. }\end{array}$ \\
$V_{O S C 1}$ & not affected & $\begin{array}{c}\text { Charge } \\
\text { injection }\end{array}$ & $\begin{array}{c}\text { Zero charge } \\
\text { injection } \\
\text { switches }\end{array}$ \\
$V_{O S C 2}$, & Not affected & Delay of & $\begin{array}{c}\text { No effect if } \\
\text { no mismatch, } \\
V_{O S 1}, V_{O S 2}\end{array}$ \\
$V_{O S 3}$ & $\frac{V_{O S 3}<<i_{C 1}, i_{C 2}}{R_{E}}$ & and $t_{d} \ll T$ \\
$I_{B 3}$ & $I_{B 3}<<i_{C 1}, i_{C 2}$ & $\begin{array}{c}\text { Delay of } \mathrm{OC}_{1} \\
\text { and } \mathrm{OC}_{2}\end{array}$ & $\begin{array}{c}\text { No effect if } \\
\text { no mismatch }\end{array}$ \\
\hline \hline
\end{tabular}

\section{EXPERIMENTAL Setup AND Results}

The prototype of the proposed CPC was realized using the components specified in Table II, according to the circuit diagram in Fig. 1, powered from a $\pm 10 \mathrm{~V}$ supply, and tested using the experimental setup shown in Fig. 5. As a proof-ofconcept, the prototype was implemented with discrete components and ICs and, hence, has not been optimised for low power consumption. The power consumption of the opamps, comparators, and switches were $P_{\mathrm{OA}} \sim 27 \mathrm{~mW}, P_{\mathrm{OC}}=102 \mathrm{~mW}$, and $P_{\mathrm{SW}}=14.8 \mathrm{~mW}$, respectively. This leads to a total power consumption $<150 \mathrm{~mW}$. The sinusoidal AC signal and the triangular reference signal in the prototype were generated by a function generator. In a real application, these signals could be generated through either a microcontroller with an embedded DAC or a waveform generator chip, for instance, the AD9837 from Analog Devices. This is a low-cost (1\$), low-power (4 $\mathrm{mA}$ ) and programmable solution that can provide an output signal (sinusoidal or triangular) with a very low thermal sensitivity and very low harmonic distortion (-68 dB).

To test the prototype for known capacitance values, two identical standard variable capacitance boxes, with negligible leakage conductance were selected. These are used to represent $C_{1}$ and $C_{2}$. To correct for the effect of bias current of opamps, a $10 \mathrm{M} \Omega$ resistor was introduced across $C_{F 1}$ and $C_{F 2}$. To smoothen output of $\mathrm{OA}_{3}$, a Butterworth low pass filter with a corner frequency of $100 \mathrm{~Hz}$ was introduced between the output

\begin{tabular}{cc||cc}
\multicolumn{4}{c}{ TABLE II } \\
\hline \multicolumn{4}{c}{ PROTOTYPE } \\
\hline \hline Component & Part/Value & Component & Part/Value \\
\hline \hline$v_{S}$ & $5 \mathrm{~V}_{\mathrm{pp}, \text { Using }}$ & $f_{S}$ & $500 \mathrm{~Hz}$ \\
& AFG3022B & & \\
$v_{t r i}$ & From AFG3022B & $f_{\text {tri }}$ & $10 \mathrm{kHz}$ \\
$S_{1}-S_{4}$ & $\mathrm{MAX} 4709$ & $V_{p}$ & $5 \mathrm{~V}$ \\
$C_{1}, C_{2}$ & $250 \mathrm{pF} \pm 50 \mathrm{pF}$ & $O A_{1}$ to $O A_{3}$ & $\mathrm{OP} 07$ \\
$O C_{1}, O C_{2}$ & $\mathrm{LM} 311$ & $C_{F 1}=C_{F 2}$ & $1 \mathrm{nF}$ \\
$R_{1}, R_{2}$ & $100 \mathrm{k} \Omega$ & $C_{F 3}$ & $1 \mathrm{nF}$ \\
\hline \hline
\end{tabular}

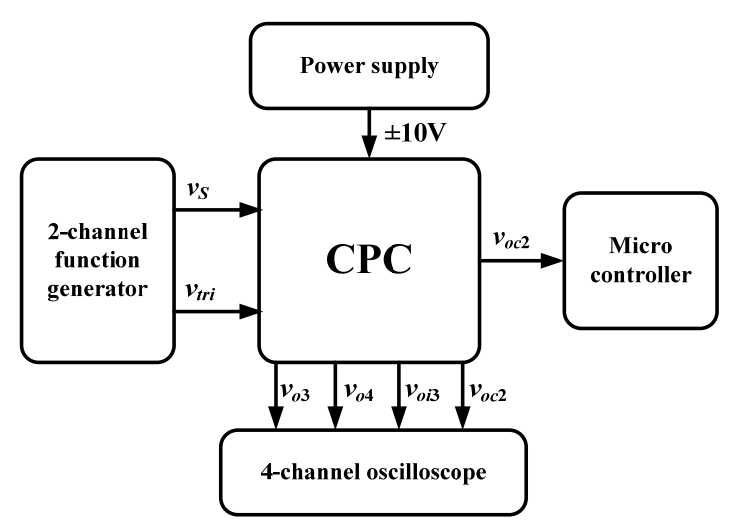

Fig. 5. Experimental setup to characterize the performance of the proposed CPC.

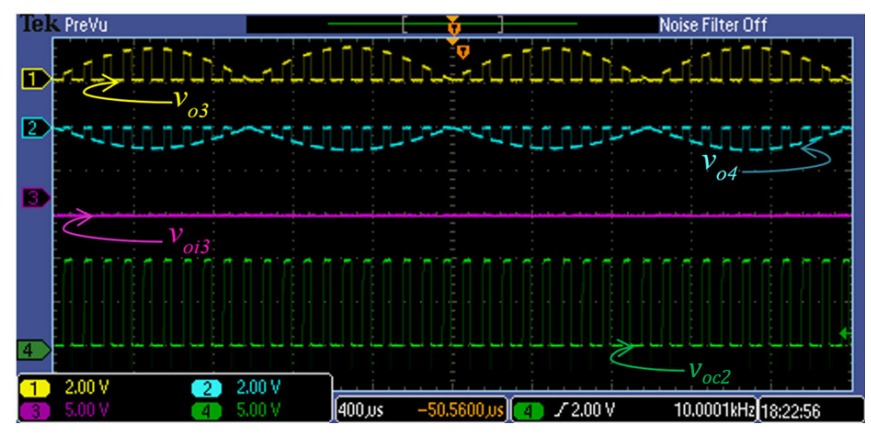

Fig. 6. Important waveforms from the prototype captured using the oscilloscope. The waveforms of $v_{03}, v_{04}, v_{o i 3}$ and $v_{o c 2}$, correspond to $C_{1}=300 \mathrm{pF}$ and $C_{2}=200 \mathrm{pF}$.

of $\mathrm{OA}_{3}$ and non-inverting terminal of $\mathrm{OC}_{2}$. During the experiments, the important waveforms from the prototype were observed using a digital oscilloscope. A screenshot of the same, corresponding to $C_{1}=300 \mathrm{pF}$ and $C_{2}=200 \mathrm{pF}$, is shown in Fig. 6. The signals $v_{o 3}, v_{o 4}, v_{o i 3}$ and $v_{o c 2}$ are indicated in Fig. 6. Since $C_{1}>C_{2}$, the amplitude of the $v_{o 3}$ is higher than that of $v_{O 4}$. This causes $v_{o i 3}$ to be negative and thus, $T_{O N}<T_{O F F}$.

\section{A. Testing the Linearity}

In order to test the linearity of the proposed scheme, $C_{1}$ and $C_{2}$ were initially set to the nominal capacitance value of $250 \mathrm{pF}$. Then, $C_{1}$ was varied for a range of $\pm 50 \mathrm{pF}$, in steps of $10 \mathrm{pF}$, while $C_{2}$ was varied by the same amount in opposite direction. For each set of values of $C_{1}$ and $C_{2}, T_{O N}$ and $T_{O F F}$ were measured using the 24-bit timer module of the ATSAM3X8E microcontroller, from Atmel, with a timing resolution of $12 \mathrm{~ns}$. This was also simultaneously measured and compared using an oscilloscope. The duty cycle, $D$, in each case was determined. The plot of $\frac{\left(T_{O F F}-T_{O N}\right)}{\left(T_{O F F}+T_{O N}\right)}$ and $\frac{\left(C_{1}-C_{2}\right)}{\left(C_{1}+C_{2}\right)}$ is given in Fig. 7. The relationship between these two, observed experimentally, is highly linear, with a maximum non-linearity error (NLE) of $0.64 \%$ FS, as can be seen in Fig. 7. In addition, the prototype circuit has a sensitivity, defined as $\left(T_{O F F}-T_{O N}\right) /\left(C_{1}-C_{2}\right)$, of $0.2 \mu \mathrm{s} / \mathrm{pF}$, which can also be obtained theoretically from (9) as $T / 2 C_{0}$. The same test was conducted for sensors possessing inverse characteristic as in (2). It was also found to be linear as expected. 


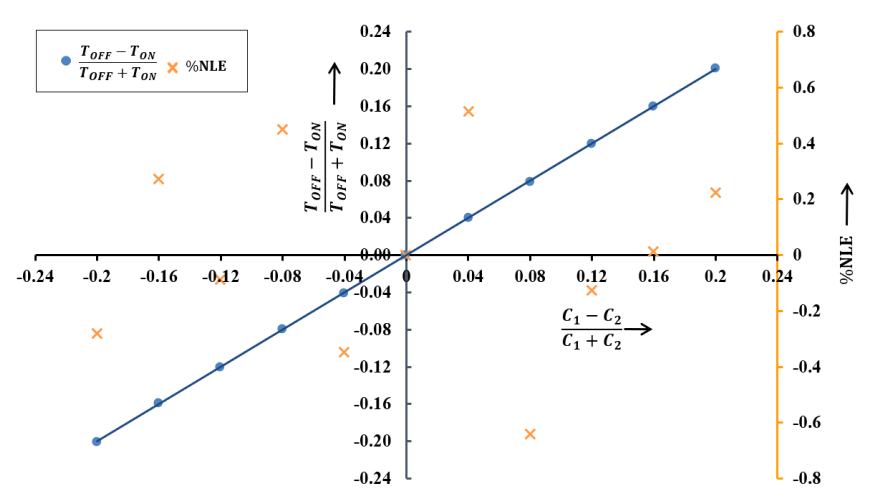

Fig. 7. Results from the prototype. The ratiometric output and the corresponding percentage non-linearity error (NLE \%) are shown.

\section{B. Testing the SNR, Resolution, and Repeatability}

To ascertain the signal-to-noise ratio (SNR), the resolution in terms of effective number of bits (ENOB) and the repeatability, first $C_{1}$ and $C_{2}$ were set to $C_{1}=200 \mathrm{pF}$ and $C_{2}=300 \mathrm{pF}$. Then, the output from the prototype was recorded for 100 consecutive measurements, all the while keeping $C_{1}$ and $C_{2}$ unchanged. The performance parameters were calculated from the recorded values using the formulae [31] given in Table III. The results obtained are presented in the same table. As can be seen, an effective resolution higher than 13 bits was obtained, which is high enough for many industrial and laboratory applications. Taking into account that the sensitivity is $0.2 \mu \mathrm{s} / \mathrm{pF}$ and that the main uncertainty source in the time-interval measurements is the timer quantization (12 ns), the resolution of the circuit prototype is $60 \mathrm{fF}$.

\section{Rise Time}

In order to determine the rise time of the proposed $\mathrm{CPC}$, an additional capacitor, $C_{\text {new }}=20 \mathrm{pF}$, was introduced. An SPDT switch $\mathrm{S}_{5}$ was used to switch $C_{n e w}$ between $C_{1}$ and $C_{2}$. One of the ends of $C_{n e w}$ was connected to node $q$ of the circuit in Fig. 1, while the other end to the common terminal of $\mathrm{S}_{5}$. The position- 0 of $\mathrm{S}_{5}$ connected $C_{n e w}$ to node $p$, whereas position-1 to node $r . \mathrm{S}_{5}$ was controlled using a switching signal $v_{s w}$, which was a $5 \mathrm{Vpp}$ square pulse signal at a frequency of $5 \mathrm{~Hz}$. For the test, $C_{2}$ and $C_{1}$ were set to $210 \mathrm{pF}$ and $270 \mathrm{pF}$, respectively. At position- 0 of $\mathrm{S}_{5}$, the resulting capacitances were $C_{2}=210 \mathrm{pF}$ and $C_{1}=290 \mathrm{pF}$, whereas at position- $1, C_{2}=(210+20) \mathrm{pF}$ and $C_{1}=(290-20) \mathrm{pF}$, introducing a $\Delta C=20 \mathrm{pF}$ in both sensing capacitors. This causes a rise in $v_{\text {oi3 }}$. The screenshot of the

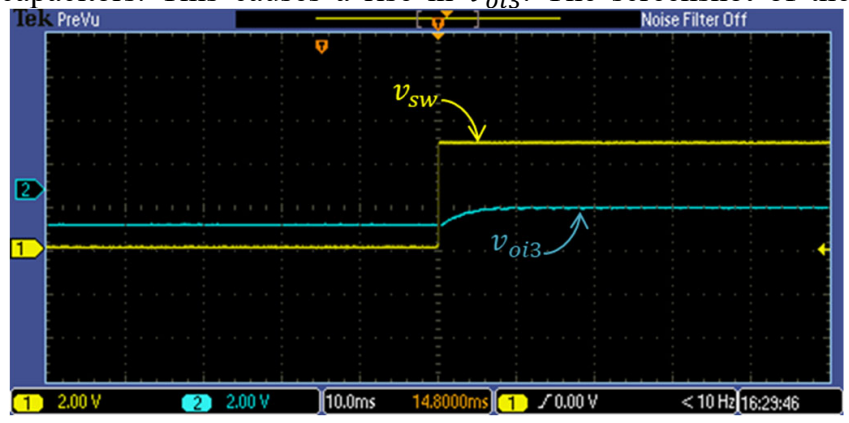

Fig. 8. Rise time test of the prototype capacitance-to-pulse-width converter.

\begin{tabular}{|c|c|c|}
\hline \multicolumn{3}{|c|}{$\begin{array}{c}\text { TABLE III } \\
\text { PROTOTYPE }\end{array}$} \\
\hline Parameter & Formula & Value \\
\hline $\begin{array}{l}\text { Signal-to-noise } \\
\text { ratio (SNR) }\end{array}$ & $S N R=10 \log \frac{\sum_{i=1}^{M} X(i)^{2}}{\sum_{i=1}^{M}[X(i)-\bar{X}]^{2}}$ & $80.12 \mathrm{~dB}$ \\
\hline $\begin{array}{l}\text { Resolution } \\
\text { (ENOB), } n \text { bits }\end{array}$ & $n=\frac{(S N R-1.76)}{6.02}$ & 13.02 bits \\
\hline Repeatability, $d$ & $d=\frac{\Delta_{\max }}{k x_{u}-k x_{l}} \times 100 \%$ & $0.027 \%$ \\
\hline \multicolumn{3}{|c|}{$\begin{array}{l}X(i)=i^{\text {th }} \text { measurement, } \bar{X}=\text { average value of the measured data } \\
M=\text { total number of measurements recorded for the repeatability study, } \\
k x_{u}=\text { upper limit of measurement range of CPC, } k x_{l}=\text { lower limit of } \\
\text { measurement range of CPC, and } \Delta_{\text {rmax }}=\text { maximum difference between } \\
\text { repeated measurements }\end{array}$} \\
\hline
\end{tabular}

oscilloscope indicating $v_{s w}$ and $v_{o i 3}$ is given in Fig. 8. The time taken for $v_{o i 3}$ to rise from $10 \%$ to $90 \%$ of its final value was found to be approximately $5 \mathrm{~ms}$. The rise time could be improved by decreasing the value of $C_{F 3}$.

\section{DISCUSSION}

The proposed CPC provides a PWM signal corresponding to the change in sensor capacitance, with the measurand. The quasi-digital PWM signal is easily digitized using a counter. This approach gives a high update rate at relatively low complexity, cost, and power. The sensor is excited by a sinusoidal signal, which is inevitable in applications where measurement at particular frequency/frequencies is required to ensure functionality [25], [26], and helps the overall measurement system deliver improved performance [30]. The final linear output, derived from the PWM signal, is independent of the sensor characteristic (linear or inverse) and $C_{0}$. The output is insensitive to parasitic capacitance and has very low sensitivity to the non-idealities of the components and ICs used. The impact of component mismatch is minimized by a one-time correction. The prototype has an NLE $<0.7 \%$, a resolution of 13.02 bits (ENOB), an SNR of $80.12 \mathrm{~dB}$ and a rise time of $5 \mathrm{~ms}$. These features widen the range of sensors that can be interfaced using the CPC. For instance, the proposed CPC can be easily applied to measure liquid-level [15] and relative humidity [32] capacitive sensors that are in the range of hundreds of $\mathrm{pF}$. The design of the proposed circuit is also suitable for IC fabrication, although it requires four resistances $\left(R_{1}, R_{2}\right.$, and those two in parallel with $C_{F 1}$ and $\left.C_{F 2}\right)$ of high value that could occupy a significant layout area; if this was critical, those resistances could be placed off-chip. A comparison of the performance of the proposed circuit vis-à-vis existing interfacing circuits for capacitive sensors was conducted focusing on similar schemes and the results are presented in Table IV.

As can be seen in Table IV, there is only one scheme [36] that accepts single-element and differential capacitive sensors and provides a quasi-digital output, like the proposed CPC. But the circuit in [36] requires a relatively expensive multiplier IC for its implementation. Such ICs have limited accuracy and hence limits the overall performance. The final output depends upon the nominal capacitance, which is another demerit. Since 


\begin{tabular}{|c|c|c|c|c|c|c|c|c|c|c|}
\hline \multicolumn{11}{|c|}{$\begin{array}{c}\text { TABLE IV } \\
\text { COMPARISON } \\
\end{array}$} \\
\hline Work & Method & $\begin{array}{l}\text { Suitable to } \\
\text { interface }\end{array}$ & $\begin{array}{c}\text { Parasitic } \\
\text { capacitance }\end{array}$ & Excitation & $\begin{array}{l}\text { Conversion } \\
\text { time }\end{array}$ & $\begin{array}{l}\text { Resolut- } \\
\text { ion }\end{array}$ & $\begin{array}{l}\text { NLE } \\
(\%)\end{array}$ & $\begin{array}{l}\text { Number } \\
\text { of com- } \\
\text { ponents }\end{array}$ & $\begin{array}{l}\text { Type of } \\
\text { Output }\end{array}$ & $\begin{array}{c}\text { Output } \\
\text { sensitive } \\
\text { to } C_{0}\end{array}$ \\
\hline [33] & Dual-slope $^{(\mathrm{a})}$ & $\begin{array}{l}\text { Single } \\
\text { element }\end{array}$ & $\begin{array}{c}\text { Not } \\
\text { sensitive }\end{array}$ & $\begin{array}{l}\text { Sine } \\
\text { wave }\end{array}$ & $24 \mathrm{~ms}$ & NS & 0.27 & M & $\begin{array}{l}\text { Time-to- } \\
\text { digital }\end{array}$ & $\begin{array}{c}\text { Not } \\
\text { sensitive }\end{array}$ \\
\hline [34] & Single-slope & $\begin{array}{l}\text { Single } \\
\text { element }\end{array}$ & Sensitive & $\begin{array}{l}\text { Square } \\
\text { wave }\end{array}$ & $15 \mu \mathrm{s}$ & 13 bits & -- & $\mathrm{L}$ & $\begin{array}{l}\text { Duty } \\
\text { Cycle }\end{array}$ & Sensitive \\
\hline$[35]$ & Switch-Bridge & DCS & Sensitive & $\begin{array}{l}\text { Square } \\
\text { wave }\end{array}$ & NM & NS & NM & M & analog & Sensitive \\
\hline$[36]$ & $\begin{array}{l}\text { Relaxation } \\
\text { Oscillator and } \\
\text { multiplier IC }\end{array}$ & $\begin{array}{l}\text { Single } \\
\text { element, } \\
\text { and DCS }\end{array}$ & Sensitive & $\begin{array}{l}\text { Square } \\
\text { wave }\end{array}$ & $\sim 15 \mathrm{~ms}$ & $0.1 \mathrm{fF}$ & $0.999^{(b)}$ & $\mathrm{M} / \mathrm{H}$ & $\begin{array}{l}\text { quasi- } \\
\text { digital }^{(\mathrm{c})}\end{array}$ & Sensitive \\
\hline [37] & $\begin{array}{l}\text { Self-balancing } \\
\text { Bridge }^{(\mathrm{d})}\end{array}$ & DCS & Sensitive & $\begin{array}{c}\text { Sine } \\
\text { wave }\end{array}$ & NM & $\begin{array}{c}0.05^{(\mathrm{e})} \\
\mathrm{NM}\end{array}$ & 0.8 & $\mathrm{H}$ & analog & $\begin{array}{c}\text { Not } \\
\text { Sensitive }\end{array}$ \\
\hline [24] & $\begin{array}{l}\text { Capacitance- } \\
\text { to-Pulse-width }\end{array}$ & $\begin{array}{l}\text { Single- } \\
\text { element }\end{array}$ & $\begin{array}{c}\text { Not } \\
\text { Sensitive }\end{array}$ & $\begin{array}{l}\text { Sine } \\
\text { wave }\end{array}$ & $13 \mathrm{~ms}$ & $\begin{array}{l}13.31 \\
\text { bits }^{(g)}\end{array}$ & $0.06^{(\mathrm{h})}$ & M & $\begin{array}{l}\text { quasi- } \\
\text { digital }\end{array}$ & $\begin{array}{c}\text { Not } \\
\text { Sensitive }\end{array}$ \\
\hline $\begin{array}{l}\text { This } \\
\text { work }\end{array}$ & $\begin{array}{l}\text { Capacitance- } \\
\text { to-Pulse-width }\end{array}$ & $\begin{array}{l}\text { Single- } \\
\text { element, } \\
\text { and DCS }\end{array}$ & $\begin{array}{c}\text { Not } \\
\text { Sensitive }\end{array}$ & $\begin{array}{c}\text { Sine } \\
\text { wave }\end{array}$ & $\sim 5 \mathrm{~ms}$ & $\begin{array}{l}13.02 \\
\text { bits }^{(g)}\end{array}$ & 0.64 & M & $\begin{array}{l}\text { quasi- } \\
\text { digital }\end{array}$ & $\begin{array}{c}\text { Not } \\
\text { Sensitive }\end{array}$ \\
\hline $\begin{array}{l}\text { Notes: } \\
\text { (a) Requ } \\
\text { (e) Stanc }\end{array}$ & $\begin{array}{l}\text { Not studied. N } \\
\text { a special clock } \\
\text { deviation of } k\end{array}$ & $\begin{array}{l}\text { M-Not mea } \\
\text { and associat } \\
\text { (f) Without }\end{array}$ & $\begin{array}{l}\text { red and repc } \\
\text { d circuit. }{ }^{(b)} \mathrm{L} \\
\text { power cons }\end{array}$ & $\begin{array}{l}\text { rted. DCS-D } \\
\text { inearity coe } \\
\text { umption of }\end{array}$ & $\begin{array}{l}\text { ferential capa } \\
\text { cient } R^{2} .{ }^{(c)} \mathrm{N} \\
\text { newave and tr }\end{array}$ & $\begin{array}{l}\text { ive sensc } \\
\text { ner ratio } \\
\text { gular wa }\end{array}$ & $\begin{array}{l}\text { A-Medic } \\
\text { ratiome } \\
\text { generato }\end{array}$ & $\begin{array}{l}- \text {-Low. H } \\
\text { d) Using t } \\
\text { ENOB, }\end{array}$ & $\begin{array}{l}\text { h. } \\
\text { nultiplier } \\
\text { nge of }+/-\end{array}$ & F. \\
\hline
\end{tabular}

the output is not ratiometric, it does not have the attractive feature of being able to interface either a linear or an inverse DCS, and still deliver a linear output. Also, it is a relaxation oscillator and hence measurement at a particular frequency of interest is not feasible. Another limitation of this scheme is its sensitivity to parasitic capacitance. Although the methods in [33] and [37] use sine wave excitation, [33] is suitable only for single-element capacitive sensors. Also, it is difficult to improve the conversion time further as it relies on the dualslope technique. In addition, [33] requires a very special clock to achieve good linearity, adding to its complexity, cost, and power consumption. The method in [37] needs two multiplier ICs, owing to which its overall performance is limited. This method is suitable only for differential capacitive sensors, and the final output is analog. So, it does not possess the advantages of a quasi-digital output that the proffered circuit has. The output in [37] is sensitive to parasitic capacitance, which is another major concern. The schemes in [34] and [35] use square wave excitation. The outputs in both these schemes are sensitive to parasitic capacitance and the final outputs sensitive to the nominal value of the capacitance. [34] is useful only for single-element capacitive sensors, while [35] is suitable for sensing differential capacitance alone. Additionally, they provide only an analog output and hence need additional circuitry to be interfaced with a digital system, which will add to the cost and amount of power consumed. The circuits in [34][37] do not have insensitivity to parasitic capacitance. The proffered converter achieves parasitic insensitivity through its simple design. The circuit in [24] is a CPC where an initial study about its feasibility for a single-element capacitive sensor is reported. It is compatible with single-element sensors with linear characteristic alone, and not suitable for DCS.

\section{CONCLUSION}

The conception and realization of a new capacitance-topulse-width converter that is compatible with both singleelement and differential capacitive sensors has been presented in this paper. It uses sinusoidal excitation and provides a linear output. The output from the converter is independent of the nominal capacitance, and sensor characteristic; the sensor characteristic can be either linear or inverse. Further, it is insensitive to parasitic capacitance and has only negligible impact due to non-idealities such as offset voltage, bias current, charge injection and $\mathrm{ON}$ resistance, provided the components and ICs are selected as per the guidelines given in the paper. In the proposed scheme, the pulse-width converter part of the circuit processes the signals due to the change in the sensor capacitance alone. Hence its full capacity is used to process the most useful information; not the unwanted signal due to the offset capacitance. The prototype of the proposed CPC exhibited a maximum non-linearity error of less than $0.7 \%$, a resolution of 13.02 bits (ENOB), and an SNR of $80.12 \mathrm{~dB}$. It has a fast response indicated by a rise time of $5 \mathrm{~ms}$. All these features make the proposed CPC suitable for a wide range of applications. It is noteworthy that many capacitive-based applications employ sinusoidal AC excitation, and the proposed first of its kind CPC, with its quasi-digital PWM output, offers an effective solution to realize the digitization of the capacitive sensor output in these applications.

\section{ACKNOWLEDGMENT}

The authors would like to thank the Department of Science and Technology (DST), Govt. of India, for its financial assistance (Grant Number SERB/F/4573/2016-17) in carrying out the research activities presented in this paper. 


\section{REFERENCES}

[1] L. K. Baxter, Capacitive Sensors. Design and Applications. NewYork: IEEE Press, 1997.

[2] B. Yang, Y. F. Lu, P. H. Hu, W. Gang, J. X. Xu, Z. Tao, et al., "Absolute Position Sensing Based on a Robust Differential Capacitive Sensor with a Grounded Shield Window", Sensors, vol. 16, pp. 680, 2016.

[3] R. E. Mallory, "Method and apparatus for emulation of a linear variable differential transducer by a capacitive gaging system." U.S. Patent No. 5,708,368. 13 Jan. 1998.

[4] C. Chan, S. Lo, Y. Huang, M. Wu, M. Wang and W. Fang, "Poly-Si Based Two-Axis Differential Capacitive-Sensing Accelerometer," IEEE Sens. J., vol. 12, no. 12, pp. 3301-3308, Dec. 2012.

[5] R. A. Brookhuis, T. S. J. Lammerink and R. J. Wiegerink, "Differential capacitive sensing circuit for a multi-electrode capacitive force sensor", Sens. Actuator A Phys., vol. 234, pp. 168-179, Oct. 2015.

[6] M. I. Tiwana, A. Shashank, S. J. Redmond and N. H. Lovell, "Characterization of a capacitive tactile shear sensor for application in robotic and upper limb prostheses", Sens. Actuator A Phys., vol. 165, no. 2, pp. 164-172, 2011.

[7] T. Schlegl and H. Zangl, "Sensor interface for multimodal evaluation of capacitive sensors", J. Phys. Conf. Ser., vol. 450, no. 1, 2013.

[8] A. M. Akour, "Capacitive sensor array for nondestructive evaluation applications", M.S. thesis, Iowa State University, 1997, Accessed on: May, $24^{\text {th }} 2020$.

[9] G. Singh, A. Nelson, S. Lu, R. Robucci, C. Patel and N. Banerjee, "EventDriven Low-Power Gesture Recognition Using Differential Capacitance," IEEE Sens. J., vol. 16, no. 12, pp. 4955-4967, June 15, 2016

[10]E. Ghafar-Zadeh, M. Sawan, V. P. Chodavarapu and T. Hosseini-Nia, "Bacteria Growth Monitoring Through a Differential CMOS Capacitive Sensor," IEEE Trans Biomed Circuits Sys, vol. 4, no. 4, pp. 232-238, Aug. 2010.

[11] S. T. Moe, K. Schjølberg-Henriksen, D.T. Wang, E. Lund, J. Nysæther, L. Furuberg, M. Visser, T. Fallet, R. W. Bernstein, "Capacitive differential pressure sensor for harsh environments", Sens. Actuator A Phys., vol. 83, no. $1-3$, pp. 30-33, 2000.

[12] J. Huang, K. Chen and C. Chien, "A differential capacitive sensing circuit for micro-machined omnidirectional microphone," 6th IEEE International Conference on Nano/Micro Engineered and Molecular Systems, Kaohsiung, 2011, pp. 948-951.

[13]B. George and V. J. Kumar, "Analysis of the Switched-Capacitor DualSlope Capacitance-to-Digital Converter," IEEE Trans Instrum Meas, vol. 59, no. 5, pp. 997-1006, May 2010.

[14]F. Han, Z. Gao, and Y. Wang, "A differential capacitance to voltage converter for electrostatic levitation applications," Sens. Actuator A Phys. vol. 99, no. 3, pp. 249-255, Jun. 2002.

[15]A. Qurthobi, R. F. Iskandar, A. Krisnatal, "Design of capacitive sensor for water level measurement", J Phys Conf Ser, vol. 776, no. 1, p. 012118 , Nov. 2016.

[16]G. Scotti, S. Pennisi, P. Monsurrò, A. Trifiletti, "88- $\mu$ A 1 MHz StrayInsensitive CMOS Current-Mode Interface IC for Differential Capacitive Sensors," IEEE Trans Circuits Syst I Regul Pap, vol. 61, no. 7, pp. 1905 1916, July 2014.

[17] S. Wang, T. J. Koickal, A. Hamilton, E. Mastropaolo, R. Cheung, A. Abel, L. S. Smith, and L. Wang, "A power-efficient capacitive read-out circuit with parasitic-cancellation for MEMS cochlea sensors," IEEE Trans. Biomed. Circuits Syst., vol. 10, no. 1, pp. 25-37, Feb. 2016

[18] E. Sisinni, A. Depari, A. Flammini, G. Ferri, V. Stornelli and G. Barile, "Full-analog parasitic capacitance compensation for AC-excited differential sensors," IEEE Trans Instrum Meas, vol. 69, no. 8, pp. 58905899, Aug. 2020.

[19]P. Bruschi, N. Nizza and M. Dei, "A low-power capacitance to pulse width converter for MEMS interfacing," ESSCIRC 2008 - 34th European SolidState Circuits Conference, Edinburgh, 2008, pp. 446-449.

[20]F. Aezinia and B. Bahreyni, "Low-power, parasitic-insensitive interface circuit for capacitive microsensors," IET Circuits, Devices \& Sys., vol. 10, no. 2 , pp. 104-110, 2016

[21]L. Areekath, B. George and F. Reverter, "An Extended Study on an Interference-Insensitive Switched Capacitor CDC," IEEE Sens. J., vol. 19 no. 18 , pp. 8283-8292, Sept.15, 2019.

[22]N. Nizza, M. Dei, F. Butti and P. Bruschi, "A Low-Power Interface for Capacitive Sensors With PWM Output and Intrinsic Low Pass Characteristic," IEEE Trans Circuits Syst I Regul Pap, vol. 60, no. 6, pp.
1419-1431, June 2013

[23]F. Reverter, "The art of directly interfacing sensors to microcontrollers", $J$. Low Power Electron. Appl., vol. 2, no. 4, pp.265-281, Dec. 2012.

[24]L. Areekath, B. George, and F. Reverter, "A Closed-Loop Capacitance to Pulse-Width Converter for Single Element Capacitive Sensors", 13th IEEE International Conference on Sensing Technology (ICST), Sydney, Australia, Dec. 2019, pp. 89-92.

[25] C. Baby K. and B. George, "A capacitive ice layer detection system suitable for autonomous inspection of runways using an ROV," 2012 IEEE International Symposium on Robotic and Sensors Environments Proceedings, Magdeburg, 2012, pp. 127-132.

[26]E. L. Tan, W. N. Ng, R. Shao, B. D. Pereles, K. G. Ong, "A wireless, passive sensor for quantifying packaged food quality", Sensors, vol. 7, no. 9, pp.1747-56, Sept. 2007.

[27] S. C. Bera and H. Mandal, "A Flow Measurement Technique Using a Noncontact Capacitance-Type Orifice Transducer for a Conducting Liquid," IEEE Trans Instrum Meas, vol. 61, no. 9, pp. 2553-2559, Sept. 2012.

[28]D. Goeger, M. Blankertz and H. Woern, "A tactile proximity sensor," IEEE SENSORS 2010, Kona, HI, 2010, pp. 589-594.

[29]J. Das, S. Dey, S. M. Hossain, Z. M. C. Rittersma and H. Saha, "A hygrometer comprising a porous silicon humidity sensor with phasedetection electronics," IEEE Sens. J., vol. 3, no. 4, pp. 414-420, Aug. 2003.

[30]R. Pallas-Areny, J. G. Webster, "Sensors and signal conditioning", John Wiley \& Sons; Nov., 2012.

[31]L. Xu, S. Sun, Z. Cao, W. Yang, "Performance analysis of a digital capacitance measuring circuit”, Rev. Sci. Instrum., vol. 86, no. 5, p. 054703 .

[32]F. Reverter and Ò. Casas, "Direct interface circuit for capacitive humidity sensors," Sens. Actuators A, vol. 143, pp. 315-322, 2008.

[33]P. Vooka and B. George, "An Improved Capacitance-to-Digital Converter for Leaky Capacitive Sensors," IEEE Sens. J., vol. 15, no. 11, pp. 62386247, Nov. 2015.

[34]Z. Ignjatovic and M. F. Bocko, "An interface circuit for measuring capacitance changes based upon capacitance-to-duty cycle (CDC) converter," IEEE Sens. J., vol. 5, no. 3, pp. 403-410, June 2005.

[35]X. Ding, K. Zhu and H. Li, "A Switch-Bridge-Based Readout Circuit for Differential Capacitance Measurement in MEMS Resonators," IEEE Sens. J., vol. 17, no. 21, pp. 6978-6985, 1 Nov.1, 2017.

[36] A. De Marcellis, C. Reig and M. D. Cubells-Beltran, "A Capacitance-toTime Converter-Based Electronic Interface for Differential Capacitive Sensors", Electronics, vol. 8, no. 1, pp. 80, Jan. 2019.

[37] A. Depari, E. Sisinni, A. Flammini, G. Ferri, V. Stornelli, G. Barile, F. R. Parente, "Autobalancing Analog Front End for Full-Range Differential Capacitive Sensing," IEEE Trans Instrum Meas, vol. 67, no. 4, pp. 885893, April 2018. 